\title{
The Diffuse Interstellar Bands: A Major Problem in Astronomical Spectroscopy
}

\author{
Peter J. Sarre \\ School of Chemistry, The University of Nottingham, University Park, Nottingham, \\ NG7 2RD, United Kingdom
}

\begin{abstract}
A critical review of the very long-standing problem of the diffuse interstellar bands is presented with emphasis on spectroscopic aspects of observational, modelling and laboratory-based research. Some research themes and ideas that could be explored theoretically and experimentally are discussed. The article is based on the Journal of Molecular Spectroscopy Review Lecture presented at the 60th Ohio State University International Symposium on Molecular Spectroscopy, June 2005.
\end{abstract}

Key words: diffuse interstellar band, molecular spectroscopy, DIB, modelling, dust, Red Rectangle

\section{Introduction and a brief history}

The longest standing challenge in astronomical spectroscopy is to assign the diffuse interstellar bands. These absorption features appear in spectra recorded towards stars that lie behind diffuse interstellar clouds and fall largely in the visible part of the electromagnetic spectrum. The first observational record is that made by Mary Lea Heger during her PhD research at Lick Observatory [1] but systematic studies began with the work of Paul Merrill [2,3]. In 1934 he wrote [2]: 'Recent observations at Mount Wilson, made chiefly to investigate the interstellar sodium lines, have disclosed four additional detached lines in the yellow and red whose approximate wave-lengths are 5780.4, 5796.9, 6283.9, and $6613.9 \AA$, respectively. These lines, found in types Oa to A4, behave like interstellar lines with regard to occurrence, intensity, and displacement. Instead of being narrow and sharp, however, as interstellar lines should be, they are somewhat widened and have rather diffuse edges. Their chemical identifications have not been found. The widening of the lines and the difficulty of identification make the problem of their atomic origin an interesting one.' 
Merrill's studies predated by a few years the discovery of the first interstellar molecules, namely $\mathrm{CH}, \mathrm{CH}^{+}$and $\mathrm{CN}$, that was made possible through a combination of observational and laboratory spectroscopy as described by Herzberg [4].

The early diffuse band researchers would surely not have thought that the solution to the diffuse band problem would still be so elusive. It is a particularly remarkable situation given the huge scientific and technological advances made over many decades. This includes the development of radio and infrared astronomy that has allowed the detection of over one hundred molecules in interstellar clouds. However, the spectral characteristics of the bands are now much better defined and this has aided progress, most notably through stimulating laboratory research and in ruling out various suggested potential carriers. A major difficulty remains in deciding which laboratory and theoretical experiments should be performed and this is where further selected observations and spectral modelling can contribute.

A major article was published by George Herbig in 1975 and includes figure 1. which illustrates the principal characteristics of the interstellar (IS) bands [5]. Spectra recorded towards the star HD 183143 which is reddened by intervening dust and an unreddened reference standard are shown with some of the key bands indicated, viz. $\lambda \lambda 5780,5797$ and 5849 (upper), together with $\lambda 6195$ and $\lambda 6177$ (lower). The bands widths differ in figure 1, showing narrow $(\lambda 6195)$, intermediate $(\lambda 5780)$ and broad $(\lambda 6177)$ examples. They are described as being 'diffuse' as their widths are greater than those of absorption features of known atoms and molecules along the line of sight. Described sometimes as fine structure on the interstellar extinction curve, this aspect is illustrated in figure 2, again for HD 183143, which appeared in a second key review published by Herbig twenty years later [6]. A number of other articles have been written covering diffuse band research $[7,8,9,10,11,12]$ as well as a new general review of diffuse cloud chemistry and spectroscopy [13]. Data for a substantial part of the spectrum are summarized in figure 3.

In this paper the very broad $2175 \AA$ absorption feature, which may be related to the diffuse band problem, is not discussed. Also only brief reference is made to the 'unidentified' infrared bands though their carriers are quite possibly related to the diffuse band issue. I concentrate on the problem as viewed from a spectroscopic perspective, describe recent results, and suggest some new areas that may help in the search for an assignment. 


\section{Characteristics of the diffuse interstellar band spectrum}

Over three hundred diffuse interstellar bands have been documented, the most striking characteristic being their widths which range between $\sim 2$ and $\sim 100 \mathrm{~cm}^{-1}$ [6]. Their diffuseness is most commonly attributed to short lifetimes of the excited states of the transitions as discussed by Smith et al. [7]. That the bands arise in interstellar rather than stellar or circumstellar material is beyond doubt at least for those cases where this has been investigated. In a type of study undertaken by Merrill [3], observations towards the binary HD 23180 on successive observing nights (figure 4.) show that the diffuse bands, together with interstellar sodium, are 'stationary' and do not share the Doppler shift of stellar features such as the $\lambda 5875$ line of photospheric Helium [15]. The word 'band' is commonly used, although with the exception of some data from high-resolution studies there is little evidence that the widths are due to the unresolved blending of individual rotational lines as could arise in the electronic spectrum of a gas-phase molecule. The spectra are confined to the range between $\sim 4000$ and $\sim 13,000 \AA$ which is roughly equivalent to photon energies in the $1-3 \mathrm{eV}$ range. The carriers are ubiquitous, being found in numerous reddened Galactic lines of sight and also in external galaxies including the Magellanic clouds [16,17,18,19], starburst galaxies [20], NGC 1448 [21] and a damped Ly $\alpha$ system at $z_{a}=0.524$ [22].

There is a good correlation between the band strengths and the reddening index $E_{B-V}$. This indicates that the dust particles that cause optical extinction are probably associated with the diffuse band carriers although these relatively large micron-sized grains are very unlikely to be directly responsible for the diffuse band absorptions. The extent of the correlation for $\lambda 5780$ is illustrated in figure 5, [23] which also highlights the real scatter that exists, the origin of which is not understood. Examples where this general relationship with extinction does not appear to hold well are rare but significant [24]. A second important correlation is that with the column density of hydrogen atoms rather than with molecular hydrogen [25]

Key spectroscopic characteristics include a lack of regularity in the wavenumbers of the bands, as might be expected to be present in the electronic spectrum of a molecule, and a lack of common band widths as would occur if the same excited state with a short lifetime were accessed by more than one transition. However, if all optical excitations originate in the same quantum level on account of the low temperature of the cloud, then appearance of common widths is unlikely. The breadth of the bands has long been recognised as an extraordinary property which is considered by many to be a defining characteristic and one that might be expected to play a major role in finding a solution. Given the generally good correlation with $E_{B-V}$, this led to proposals based on optical absorptions of carriers that are in or on grains. In this 
case the widths are generally attributed to broadening of a transition due to the condensed phase environment. However, the alternative proposal that the bands originate from electronic transitions in gas-phase molecules has gained ground particularly over the last c. 25 years. A key aspect is the recognition that large molecules can accommodate photon absorption without destruction and give rise to broad spectra due to fast internal relaxation processes [7].

There is a wide range of data available from both photographic [5,26] and CCD surveys with those up to the year 2000 described by Tuairisg et al. [14], to which further studies have been added $[27,28]$.

\section{Proposals for the origin of the diffuse bands}

The idea of molecules being responsible for the bands stretches back to the 1930s, followed by a period in which a dust grain origin was preferred. A molecular origin was championed for many years by Herzberg, although the specific idea of predissocation or preionisation being responsible for the widths is not now favoured. Suggestions for assignments include colour centres, lattice defects, the hydrogen anion, porphyrins, carbon chains, charge transfer transitions and molecular hydrogen. To this list of possibilities polycyclic aromatic hydrocarbons, simple or encapsulated fullerenes and carbon nanotubes may be added. It is of interest that the discovery of $\mathrm{C}_{60}$ was made in experiments motivated in part by the search for a solution to the diffuse band problem. The full history has been reviewed in a number of papers $[5,6,8,9,11]$.

The discovery of widespread 'unidentified' infrared (UIR) emission bands arising from polycyclic aromatic hydrocarbons (PAHs) in nebular and other regions (and also recently in the general interstellar medium) has led to the development of the 'PAH hypothesis' $[29,30]$ and a huge laboratory and theoretical effort focussed on PAHs as possible carriers [31,32,33,34].

\section{Spectroscopic aspects: the molecular hypothesis}

This section is written under the assumption that the diffuse bands are caused by electronic transitions of gas-phase molecules which under interstellar conditions have low internal temperatures. It may be assumed that all molecules will be in their lowest vibrational state, with the rotational temperature ranging between about $3 \mathrm{~K}$ for a polar molecule as found for $\mathrm{CN}$ and c. $100 \mathrm{~K}$ as for $\mathrm{C}_{2}$ in some lines of sight. The shapes and strengths of the diffuse bands are many and varied, ranging from narrow bands with asymmetry and substructure such as $\lambda \lambda 5797,6614$ and $6376 / 6379$, to the broadest and strongest 
$\lambda 4428$ (formerly $\lambda 4430$ ) band which exhibits no fine structure even observed with $10^{6}$ resolving power [35]; it obeys a perfect fit to a Lorentzian function [36].

\subsection{Regularities and correlations}

Given the importance of pattern recognition in spectroscopic assignments, attempts have been made to find links between bands. Herbig [37] noted a small set of bands near $6800 \AA$ with intervals of $\sim 35 \mathrm{~cm}^{-1}$ and possible assignments in terms of $\mathrm{C}_{n} \mathrm{H}_{2}$-type polyene molecules have been offered $[38,39]$. The core idea is that the bands are $K$ sub-bands and the separations of $\sim 35 \mathrm{~cm}^{-1}$ correspond to $\sim 4 A$ where $A$ is the rotational constant associated with the $a$ inertial axis $[38,39]$. However, only those sub-bands with a statistical weight of 3 are clearly observed so there remains some doubt as to whether this is the correct explanation [40]. A set of possibly linked bands has been put forward by Weselak et al. [41] who tentatively suggested that $\lambda \lambda 5809,6196,6614$ and 6660 might be from the same carrier but this awaits further study.

Numerous investigations of possible correlations between the strengths of various bands have been made, resulting in the concept of 'families' first described by Krełowski and Walker in 1987 [42] and since developed by others (see [43] for a summary). One case where there is a particularly good correlation is that between $\lambda 6614$ and $\lambda 6196$ which has been found to have a correlation coefficient in equivalent width of $0.98 \pm 0.18$ over 62 lines-of-sight [44]. Following a detailed statistical assessment a value of 0.985 was reported at the Symposium based on APO survey results [45]. Even in this case the question as to whether the two bands arise from the same carrier remains open; from high resolution and high signal-to-noise spectra Galazutdinov et al. have drawn the conclusion that the ratio of equivalent widths of the two diffuse bands is not always exactly the same [46].

A number of weak diffuse bands appear to be correlated with the presence of $\mathrm{C}_{2}$ [47] which suggests that the formation chemistry for these band carriers may be similar to that of $\mathrm{C}_{2}$ (and $\mathrm{C}_{3}$ ). Some of the ' $\mathrm{C}_{2}$ ' bands occur in pairs with very similar separations of 20.7, 20.7 and $20.9 \mathrm{~cm}^{-1}$ which may arise from spin-orbit interaction. A further study of these bands has been made from which it is deduced that the ' $\mathrm{C}_{2}$ diffuse band' designation is premature and that it is unlikely that the bands are related to $\mathrm{C}_{2}$ [48]. In the event that any two diffuse bands originate from the same level of the same carrier then the correlation must of course be perfect. However, in seeking insight into the problem, there is considerable merit in finding families of bands that generally behave in a similar way. 


\subsection{High-resolution spectra and modelling}

Interest in high-resolution spectroscopy has grown since the discovery of fine structure in some of the narrower diffuse bands such as $\lambda 6614[49,50]$. The $\lambda 6614$ band has been found to have three main components that have the appearance of unresolved P, Q and R branches of an electronic transition of a large molecule [49]. Building on the principles first applied to diffuse band profiles by Danks and Lambert [51], the profile of $\lambda 6614$ was fitted in a $\chi$ squared minimisation procedure to derive molecular constants and rotational temperatures as shown in figure 6 [52]. A range of molecular geometries and types of electronic transition was explored, the best fit to the profile being achieved for an oblate symmetric top like coronene where the $\tilde{A}^{1} \mathrm{~B}_{2}-\tilde{X}^{1} \mathrm{~A}_{1}$ transition is vibronically induced as the origin band is symmetry forbidden. A plausible fit was obtained for a range of low rotational temperatures and it was noted that a $\mathrm{C}_{18}$ (monocyclic cumulenic) ring could also be a candidate [52]. Neither of these specific molecules is a diffuse band carrier as the strongest band of coronene at $4031.4 \AA$ does not appear in the spectra of diffuse clouds [53] and the $\tilde{A}^{1} \mathrm{~A}_{2}{ }^{\prime \prime}-\tilde{X}^{1} \mathrm{~A}_{1}{ }^{\prime}$ transition of $\mathrm{C}_{18}$ occurs at $5928.5 \AA$ which is also not detected [54]. However, this modelling study did illustrate the principle that the fine structure could be reproduced in terms a molecular electronic transition and at typical interstellar temperatures. An ultra-high resolution spectrum of $\lambda 5797$ has revealed an even higher level of fine structure [55] but this has not yet been modelled successfully. A rough estimate of the size of carrier can be made by taking the separation of the ' $\mathrm{P}$ ' and ' $\mathrm{R}$ ' branch peaks. Using this approach Ehrenfreund and Foing inferred that the spectra of $\lambda \lambda 6614,5797$ and 6379 were consistent with PAH molecules with more than 40 carbon atoms, chains of $\sim 12-18$ carbon atoms, rings with 30 carbons or fullerenes [50]. Schulz et al. have suggested that a polyene with 9-13 carbon atoms could account for the $\lambda 6614$ fine structure [39]. All of these approaches assume thermally populated rotational levels. A more detailed treatment of level populations has been presented by Mulas [56]. In a study of the $\lambda \lambda 6196$ and 6379 bands, it was reported that rotational contour models did not fit either profile well, there being more structure in the model than is observed [57]. The level of agreement was improved by invoking a broadening of $\sim 0.2-0.3$ $\mathrm{cm}^{-1}$ [57]. In related work, contour modelling predictions have been pursued for PAH-type systems and fullerenes [58,59] and spectra of the $\lambda 5850$ band and nearby features have been discussed in terms of possible rotational branch structure [60].

A growing area of observational research is the study of profile variation that might be indicative of changes in level populations, physical state or local interstellar conditions. Neglecting band broadening that occurs simply because there are two or more clouds in a line-of-sight e.g. HD 183143 [61], there are reports of unusual band widths and shifts in spectra taken towards Orion stars 
[63,62] and HD 34078 [64]. The origin of this effect is not established.

\subsection{Carbon isotope structure}

A completely different hypothesis for the origin of the 'triplet' structure of the $\lambda 6614$ band has been put forward by Webster [65]. It is suggested that each of the component peaks arises from a separate ${ }^{13} \mathrm{C}$ isotopic modification of the same molecule where the relative positions of the components are determined by a vibrational isotope shift in the excited electronic state. The work highlights the importance of considering the ${ }^{13} \mathrm{C}$ isotope, provides a good fit to the spectrum and has also been applied to the $\lambda 6196$ profile in connection with the zero-point vibrational isotope shift [66]. Further observations of $\lambda \lambda 5797$ and 6614 have been taken as evidence in support of carbon isotope structure in the spectrum [67]. However, in a recent study of $\lambda 6614$ at high resolution, it is reported that the positions (wavelengths) of two of the substructure peaks vary according to the line-of-sight [68]. While the wavelength of the strongest central 'Q' component does not change, the 'Q-R' and 'Q-P' separations increase and decrease in concert though to different extents. This is reminiscent of the behaviour of molecular band contours as a function of temperature and a range of $\sim 21-25 \mathrm{~K}$ for $T_{\text {rot }}$ was deduced. From these observations it was concluded that the observed variations rule out the idea that the $\lambda 6614$ substructure arises from isotope shifts [68].

\section{Circumstellar and nebular environments}

Study of the diffuse band carriers in localised astrophysical environments such as stars and nebulae offers the potential to investigate their spectroscopic transitions under different excitation conditions and potentially to learn something of their formation mechanism. Unfortunately such data are extremely rare in spite of many observational searches. The circumstellar shell of the nearby carbon star IRC $+10^{\circ} 216$ and the Red Rectangle nebula are of interest in this context.

\subsection{The circumstellar shell of IRC $+10^{\circ} 216$}

One of the more likely environments in which the diffuse band carriers might be found is in the circumstellar envelope of a mass-losing carbon star. A summary of observations made in the search for circumstellar diffuse band absorptions in carbon stars and planetary nebulae is given in references $[69,70,71]$ but with 
very largely negative or uncertain results. The most studied circumstellar shell in astrochemistry is IRC $+10^{\circ} 216$ in which numerous molecules have been detected by radio, infrared and optical spectroscopy. A search for diffuse band absorption due to carriers in the envelope was made by recording spectra towards a star (Star 6) which lies behind part of the extended shell as shown in figure 7 [72]. No absorption at the expected position for $\lambda 6614$ was found. Although the precursors to the diffuse band carriers may well be formed in such objects, it seems that further chemical or UV processing is required before they become spectroscopically active in the interstellar medium.

\subsection{The Red Rectangle}

The importance of the Red Rectangle nebula and its central binary star HD 44179 was first signalled in a paper by Cohen et al. in 1975 [73]. This biconical nebula displays many unusual unassigned spectroscopic features from the infrared to the far-UV including strong optical emission bands [74] with wavelengths which lie close to some of the diffuse bands seen in absorption $[75,76,77,78,79]$. This is illustrated in figure 8 for a small part of the region near $5800 \AA$ where the Red Rectangle spectrum at an offset from the central star of $7.5 \pm 2.5^{\prime \prime}$ is compared with the diffuse band absorption spectrum towards HD 183143. Measurements of the peak wavelengths and widths of the strongest emission bands show that they change with offset from the exciting star and that these two attributes converge towards the values seen in diffuse band absorption. However, even at the highest offset so far attained they are not coincident with the interstellar absorption data $[78,79,80,81]$. A possible explanation is that the internal temperature of the carrier is 'locked' as occurs for $\mathrm{C}_{2}$. Resonance fluorescence of the Swan $5165 \AA(0,0)$ band of $\mathrm{C}_{2}\left(\mathrm{~d}^{3} \Pi_{g}\right.$ $\left.\mathrm{a}^{3} \Pi_{u}\right)$ is detectable out to at least $13^{\prime \prime}$ offset in the nebula. This means that the metastable $\mathrm{a}^{3} \Pi_{u}$ state with $\sim 700 \mathrm{~cm}^{-1}$ excitation is significantly populated. Moreover the high-offset $\mathrm{C}_{2}$ spectrum has a band head which indicates a rotational temperature in excess of that found via the $\mathrm{C}_{2}$ Phillips $\mathrm{A}^{1} \Pi_{u}-\mathrm{X}^{1} \Sigma_{g}^{+}$ system (only) in diffuse interstellar clouds.

The shapes of two of the most prominent emission bands near 5800 and $6615 \AA$ are reminiscent of molecular rotational contours $[77,78]$ but there may also be contributions from unresolved vibrational sequence band structure [81]. The bands also appear weakly in the spectrum of the R CrB star V854 Cen at minimum light [82]. Whether the set of unidentified Red Rectangle emission bands and diffuse bands arise from the same carriers as suggested [78] will probably be proven definitively only when the carriers are identified and the spectra are reproduced in the laboratory. However the likelihood of this being the case is sufficient to have stimulated new laboratory searches for 'diffuse band' spectra in emission, but so far without success. 


\section{Spatial distribution of the diffuse band carriers}

Considerable research effort has been committed to searching for correlations between bands by recording spectra along many lines of sight through different types of clouds. In a complementary approach we have commenced a new observing program in which spectra are recorded at extremely high signalto-noise ratio towards binary stars that are separated in light path by only 100-10,000 AU. This simplifies the situation because, in contrast to widely separated unrelated lines of sight, the cloud conditions for the two lines-ofsight should have some chemical and physical similarities. However, from an observational viewpoint, discerning small differences in diffuse band strengths is very challenging and hence requires long integration times. The work is motivated in part by the subject of this article, but in addition there is much general astronomical interest in determining the level of 'small-scale structure' of the interstellar medium.

Spectra recorded using UCLES at the Anglo-Australian Telescope towards $\rho$ Oph A and $\rho$ Oph B are shown in figure 9. These stars are separated by $3.1^{\prime \prime}$ which at a distance of $\sim 120$ pc results in a sky-projected separation of $\sim 370$ AU. Taking the ratio of the spectra towards $\rho$ Oph A and B yields the lower trace of figure 9. Equivalent width measurements give quite a large increase in strength of both $\lambda 5780$ and $\lambda 5797$ of c. $5 \%$ from A to B. Initial results indicate that this type of study will produce further information on diffuse

band families, relationships between diffuse band carriers and atoms/molecules along the same lines-of-sight, and on the physical conditions in the cloud.

\section{Solving the problem}

Most researchers consider that the diffuse bands arise from a set of carbonbased absorbers with size between the dust grains that cause visual extinction and the largest carbon molecule so far identified optically in diffuse clouds, $\mathrm{C}_{3}$ $[83,84]$. In this section a brief overview of laboratory research is given followed by a few speculative reflections on the diffuse band problem. Attention is drawn to some areas that deserve further study including clues that may be available from other areas such as the UIR bands and the Red Rectangle.

\subsection{Laboratory experiments}

An enormous wealth of laboratory electronic spectra of carbon chains, PAHs and fullerenes in neutral and ionised forms has been recorded in the gaseous 
phase and in matrices at low temperature. Although not always yielding transition frequencies as precise as desirable due to uncertainty in the degree of internal excitation in the gas phase and the influence of the matrix in condensed phase work, there is little doubt in the author's view that a correspondence with any of the most prominent features in the diffuse band spectrum would be apparent. In some instances there are also uncertainties in the astrophysical data due to stellar spectral contamination and poorly defined velocity distributions in the cloud, coupled with the problem of the unknown carrier transition rest frequency. No attempt to describe this work in full is made here; comparisons between laboratory and astrophysical data can be found in $[85,86,87]$. Recent 'near-misses' or cases where the data do not allow a definitive conclusion to be made include studies of $\mathrm{C}_{14} \mathrm{H}, \mathrm{C}_{7}^{-}, \mathrm{C}_{3} \mathrm{H}_{2}^{-}$, $\mathrm{C}_{10} \mathrm{H}_{8}^{+}$and $\mathrm{C}_{5} \mathrm{H}_{5}$. The intriguing possibility of $\mathrm{C}_{60}^{+}$being responsible for two red bands awaits gas-phase spectra [88]. There is also a possible correspondence between the gas-phase origin band of $\mathrm{CH}_{2} \mathrm{CN}^{-}$and a weak diffuse band at $8037 \AA$ [89] but this needs higher quality observational spectra to determine whether predicted satellite $K$ sub-bands are present. This partial list of recently studied spectra illustrates the surge in laboratory effort stimulated by the diffuse band problem that has led to important advances in the spectroscopy of these molecules through often very challenging experiments. There do remain some areas that are virtually untouched including the laboratory electronic spectra of gas-phase carbon polyene chains, protonated, anionic and heteroatom-containing PAHs, fullerenes and carbon nanotubes.

\subsection{Heteroatoms in and on PAHs}

Almost all astrophysically oriented research on the electronic transitions of neutral and ionised PAHs considers them to contain carbon and hydrogen only. However, the rich field of colour chemistry contains hundreds of polyaromatic compounds in which nitrogen and oxygen atoms are present either on the periphery or as part of the aromatic ring system. Their inclusion is crucial to the very strong absorption and emission of dye-type molecules in the visible part of the spectrum. Both types of chemical modification of PAHs by heteroatoms are found in meteorites. To take an example, proflavine absorbs strongly in the blue region at $445 \mathrm{~nm}$ (in water at $\mathrm{pH} 7$ ) with a molar extinction coefficient of $c$. 40,000. Some plausible diffuse band carrier candidates do not require heteroatom substitution in the ring system. An example is the quinones which contain a $\mathrm{C}=\mathrm{O}$ group. In the Red Rectangle nebula, which has a mixed carbon-oxygen chemistry, a rare infrared emission band at 6.0 $\mu \mathrm{m}$ is likely due to the $\mathrm{C}=\mathrm{O}$ stretch of an oxygenated $\mathrm{PAH}$ [90]. While terrestrial dye-type molecules are relatively small, the principle should carry over

to larger PAH systems that are likely to be present in the interstellar medium and therefore warrant laboratory and theoretical study. Some aspects of the 
electronic transitions of PAHs with exo- and endohedral nitrogen have recently been considered in connection with possible diffuse band carriers [91].

\subsection{Clues from the Red Rectangle?}

The Red Rectangle displays the UIR bands strongly from both the central star and the nebula and this has led to the idea that PAHs are responsible for the unidentified optical emission bands including those close in wavelength to some diffuse bands. While there is no correlation between the $3.3 \mu \mathrm{m}$ 'PAH' emission feature and the unidentified optical red/yellow emission bands [92], blue fluorescence has been discovered [93] that does follow the $3.3 \mu \mathrm{m}$ IR emission [94]. This, with other factors, has led to the proposed presence of small gas-phase PAHs in the Red Rectangle as they are known to fluoresce in the near-UV [93,94]. However, recent results from the Spitzer satellite have shown that the carbon-oxygen chemistry in the Red Rectangle is mixed [95] with oxygen-rich material probably being present in the nebula. Hence inorganic or organometallic molecules (or dust grains) as carriers of the unidentified optical electronic emission spectra (such as in figure 8) and the 'blue fluorescence' $[93,94]$ cannot be ruled out. It is notable that a set of natural olivine samples demonstrate UV-pumped photoluminescence of hole centres in the same 'blue fluorescence' range [96] as that attributed [94] to small PAHs in the Red Rectangle. It is potentially of interest that the $5800 \AA$ bands fall very close to the 'yellow' bands of gas phase FeO. While the observed spectrum does not arise from the gas-phase iron oxide molecule, perhaps this is more than a coincidence.

\section{Acknowledgements}

That part of the work described which has been undertaken at Nottingham has been conducted in collaboration with other researchers to whom I am greatly indebted. I wish particularly to thank Paul Boichat, Martin Cordiner, Stephen Fossey, Rob Hibbins, Mark Hurst, Tim Kendall, Tom Kerr, June McCombie, Janet Miles, Jules Russell, Arfon Smith and Radmila Topalovic. The observational work described was awarded by the UK Panel for the allocation of telescope time (PATT) and the research made use of the SIMBAD database, operated at CDS, Strasbourg, France. I would like to thank an anonymous referee for helpful comments. 


\section{Glossary}

$\operatorname{arcsec}(")$ : A measure of angular separation. It is one sixtieth of an arc minute and $1 / 3600$ th of a degree.

AU: An Astronomical Unit is the mean distance between the Earth and the Sun. $1 \mathrm{AU}=1.495979 \times 10^{11} \mathrm{~m}$.

CCD: charge-coupled device.

Column density: the number of atoms, e.g. hydrogen, in a column of area (normally $1 \mathrm{~cm}^{-2}$ ) extending from the Earth to the star.

$E_{B-V}=(B-V)-(B-V)_{0}$ is the colour excess (in magnitudes - see below) due to interstellar extinction, where $B$ and $V$ are defined at 440 and $550 \mathrm{~nm}$, respectively, and $(B-V)_{0}$ is the intrinsic value for the star.

Equivalent width, $W_{\lambda}=\int_{-\infty}^{\infty} \frac{I_{0}(\lambda)-I(\lambda)}{I_{0}(\lambda)} d \lambda$. It is usually quoted in $\AA$.

Extinction: A term used to describe the amount of absorption and scattering of light by gas and dust in the interstellar medium. It is normally quoted in magnitudes.

Interstellar reddening: For a star lying behind an interstellar cloud, its blue light is more strongly attenuated by interstellar material than red light. Therefore the star appears to have a redder colour than its instrinsic output.

Magnitude: a logarithmic measure of the brightness of an object or interstellar extinction such that for two stars 1 and $2, m_{1}-m_{2}=-2.5 \times \log _{10}\left[F_{1} / F_{2}\right]$, where $F$ is the radiation flux.

$\mathrm{pc}=$ parsec: derived from parallax of one arcsec and is the distance at which two objects, separated by one AU appear to be separated by one arcsec. It is $3.085678 \times 10^{16} \mathrm{~m}$ or $\sim 3.26$ light years 


\section{References}

[1] M.L. Heger, Lick Obs. Bull. 10 (337) (1922) 146.

[2] P.W. Merrill, Publications of the Astronomical Society of the Pacific, 46 (1934) 206-207. Quotation reproduced with permission of the Editors.

[3] P.W. Merrill, Astrophys. J. 83 (1936) 126-128.

[4] G. Herzberg, J. Roy. Astron. Soc. Can. 82(3) (1988) 115-127.

[5] G.H. Herbig, Astrophys. J. 196 (1975) 129-160.

[6] G.H. Herbig, Ann. Rev. Astron. Astrophys. 33 (1995) 19-74.

[7] W.H. Smith, T.P. Snow, D.G. York, Astrophys. J. 218 (1977) 124-132.

[8] G.E. Bromage, Quart. J. Roy. Astron. Soc. 28 (1987) 294-297.

[9] Snow, T.P., in: A.G.G.M. Tielens, T.P. Snow (Eds.), The Diffuse Interstellar Bands, Kluwer, Dordrecht, 1995, pp. 325-340.

[10] D.A. Williams, Astrophysics and Space Science 237 (1996) 243-266.

[11] T.P. Snow, Spectrochim. Acta A 57 (2001) 615-626.

[12] J. Krełowski, Advances in Space Research 30 (6) (2002) 1395-1407.

[13] T.P. Snow, B.J.McCall, Ann. Rev. Astron. Astrophys. 44 (2006) 367-414.

[14] S.Ó. Tuairisg, J. Cami, B.H. Foing, P. Sonnentrucker, P. Ehrenfreund, Astron. Astrophys. Suppl. Ser. 142 (2000) 225-238.

[15] J. Krełowski, C. Sneden, Pub. Astron. Soc. Pacific 105 (1993) 1141-1149.

[16] D.H. Morgan, Quart. J. Roy. Astron. Soc. 28 (1987) 328-333, and references therein.

[17] G. Vladilo, L. Crivellari, P. Molaro, J.E. Beckman, Astron. Astrophys. 182 (1987) L59-L62.

[18] P. Ehrenfreund, J. Cami, J. Jiménez-Vicente, B.H. Foing, L. Kaper, A. van der Meer, N. Cox, L. d'Hendecourt, J.P. Maier, F. Salama, P.J. Sarre, T.P. Snow, P. Sonnentrucker, Astrophys. J. 576 (2002) L117-L120.

[19] N.L.J. Cox, M.A. Cordiner, J. Cami, B.H. Foing, P.J. Sarre, L. Kaper, P. Ehrenfreund, Astron. Astrophys. 447 (2006) 991-1009.

[20] T.M. Heckman, M.D. Lehnert, Astrophys. J. 537 (2000) 690-696, and references therein.

[21] J. Sollerman, N. Cox, S. Mattila, P. Ehrenfreund, L. Kaper, B. Leibundgut, P. Lundqvist, Astron. Astrophys. 429 (2005) 559-567, and references therein. 
[22] V.T. Junkkarinen, R.D. Cohen, E.A. Beaver, E.M. Burbidge, Lyons, R.W., G. Madejski, Astrophys. J. 614 (2004) 658-670.

[23] J. Krełowski, P. Ehrenfreund, B.H. Foing, T.P. Snow, T. Weselak, S.Ó. Tuairisg, G.A. Galazutdinov, F. Musaev, Astron. Astrophys. 347 (1999) 235-242.

[24] T.P. Snow, D.E. Welty, J. Thorburn, L.M. Hobbs, B.J. McCall, P. Sonnentrucker, D.G. York, Astrophys. J. 573 (2002) 670-677.

[25] G.H. Herbig, Astrophys. J. 407 (1993) 142-156.

[26] T.P. Snow, D.G. York, D.E. Welty, Astron. J. 82 (1977) 113-128.

[27] T. Weselak, M. Schmidt, J. Krełowski, Astron. Astrophys. Supp. 142 (2000) 239-246; 143 (2000) 542.

[28] B.J. McCall, B.L. Rachford, T.P. Snow, P. Sonnentrucker, S. Friedman, J. Thorburn, T. Oka, D.E. Welty, L.M. Hobbs, D.G. York, NASA Laboratory Astrophysics Workshop, May 1-3 2002 at NASA Ames Research Center, Moffett Field, CA 94035-1000. Publisher: NASA. Edited by Farid Salama. Reference Conference Proceedings: NASA/CP-2002-21186, 2002. p. 138.

[29] A. Léger, L. d'Hendecourt, Astron. Astrophys. 146 (1985) 81-85.

[30] G.P. van der Zwet, L.J. Allamandola, Astron. Astrophys. 146 (1985) 76-80.

[31] F. Salama, E.L.O. Bakes, L.J. Allamandola, A.G.G.M Tielens, Astrophys. J. 458 (1996) 621-636.

[32] R. Ruiterkamp, T. Halasinski, F. Salama, B.H. Foing, L.J. Allamandola, W. Schmidt, P. Ehrenfreund, Astron. Astrophys. 390 (2002) 1153-1170.

[33] J.L. Weisman, T.J. Lee, F. Salama, M. Head-Gordon, Astrophys. J. 587 (2003) 256-261, and references therein.

[34] T.M. Halasinski, F. Salama, L.J. Allamandola, Astrophys. J. 628 (2005) 555566.

[35] T.P. Snow, Astrophys. J. 567 (2002) 407-411.

[36] T.P. Snow, D. Zukowski, P. Massey, Astrophys. J. 578 (2002) 877-884.

[37] G. Herbig Astrophys. J. 331 (1988) 999-1003.

[38] R.J. Glinski, J.A. Nuth, Pub. Astron. Soc. Pac. 107 (1995) 453-461.

[39] S.A. Schulz, J.E. King, R.J. Glinski, Mon. Not. R. Astron. Soc. 312 (2000) 769-773.

[40] H. Tada et al. 'Observations Of Diffuse Interstellar Bands Near 680 nm', 60th Ohio State University International Symposium on Molecular Spectroscopy, June 2005.

[41] T. Weselak, J. Fulara, M.R. Schmidt, J. Krełowski, Astron. Astrophys. 377 (2001) 677-686. 
[42] J. Krełowski, G.A.H. Walker, Astrophys. J. 312 (1987) 860-867.

[43] N.L.J. Cox, L. Kaper, B.H. Foing, P. Ehrenfreund, Astron. Astrophys. 438 (2005) 187-199, and references therein.

[44] C. Moutou, J. Krełowski, L. d'Hendecourt, J. Jamroszczak, Astron. Astrophys. 351 (1999) 680-688.

[45] B.J. McCall et al. 'Correlations among Diffuse Interstellar Bands, Atoms, and Small Molecules', 60th Ohio State University International Symposium on Molecular Spectroscopy, June 2005.

[46] G. Galazutdinov, C. Moutou, F. Musaev, J. Krełowski, Astron. Astrophys. 384 (2002) 215-224.

[47] J.A. Thorburn, L.M. Hobbs, B.J. McCall, T. Oka, D.E. Welty, S.D. Friedman, T.P. Snow, P. Sonnentrucker, D.G. York, Astrophys. J. 584 (2003) 339-356.

[48] G.A. Galazutdinov, P. Gnacinski, I. Han, B. Lee, K. Kim, J. Krełowski, Astron. Astrophys. 447 (2006) 589-595.

[49] P.J. Sarre, J.R. Miles, T.H. Kerr, R.E. Hibbins, S.J. Fossey, W.B Somerville, Mon. Not. Roy. Astron. Soc. 277 (1995) L41-L43.

[50] P. Ehrenfreund, B.H. Foing, Astron. Astrophys. 307 (1996) L25-L28.

[51] A.C. Danks, D.L. Lambert, Mon. Not. Roy. Astron. Soc. 174 (1976) 571-586.

[52] T.H. Kerr, R.E. Hibbins, J.R. Miles, S.J. Fossey, W.B. Somerville, P.J. Sarre, Mon. Not. Roy. Astron. Soc. 283 (1996) L105-L109.

[53] P.J. Sarre, J. Chem. Soc. Farad. Disc. 109 (1998) 400.

[54] J.P. Maier, A.E. Boguslavskiy, H. Ding, G.A.H. Walker, D.A. Bohlender, Astrophys. J. 640 (2006) 369-372.

[55] T.H. Kerr, R.E. Hibbins, S.J. Fossey, J.R. Miles, P.J. Sarre, Astrophys. J. 495 (1998) 941-945.

[56] G. Mulas, Astron. Astrophys. 338 (1998) 243-261.

[57] G.A.H. Walker, A.H. Gordon, A.S. Webster, D.A. Bohlender, J. Krełowski, Astrophys. J. 561 (2001) 272-281.

[58] C. Cossart-Magos, S. Leach, Astron. Astrophys. 233 (1990) 559-569.

[59] S.A. Edwards, S. Leach, Astron. Astrophys. 272 (1993) 533-540.

[60] P. Jenniskens, I. Porceddu, P. Benvenuti, F.-X. Désert, Astron. Astrophys. 313 (1996) 649-656.

[61] G.H. Herbig, D.R. Soderblom, Astrophys. J. 252 (1982) 610-615.

[62] J. Krełowski, J. Mayo Greenberg, Astron. Astrophys. 346 (1999) 199-204.

[63] I. Porceddu, P. Benvenuti, J. Krełowski, Astron. Astrophys. 260 (1992) 391-396. 
[64] G.A. Galazutdinov, G. Manicò, J. Krełowski, Mon. Not. R. Astron. Soc. 366 (2006) 1075-1080.

[65] A. Webster, Mon. Not. Roy. Astron. Soc. 282 (1996) 1372-1380.

[66] A. Webster, Mon. Not. Roy. Astron. Soc. 349 (2004) 263-269.

[67] G.A.H. Walker, D.A. Bohlender, J. Krełowski, Astrophys. J. 530 (2000) 362-370.

[68] J. Cami, F. Salama, J. Jiménez-Vicente, G.A. Galazutdinov, J. Krełowski, Astrophys. J. 611 (2004) L113-L116.

[69] L. Začs, M.R. Schmidt, R. Szczerba, Mon. Not. Roy. Astron. Soc. 306 (1999) 903-912.

[70] L. Začs, R. Spelmanis, F.A. Raivis, G.A. Galazutdinov, Mon. Not. Roy. Astron. Soc. 339 (2003) 460-466.

[71] N. Mauron, T.R. Kendall, Astron. Astrophys. 428 (2004) 535-543.

[72] T.R. Kendall, N. Mauron, J. McCombie, P.J. Sarre, Astron. Astrophys. 387 (2002) 624-634.

[73] M. Cohen, C.M. Anderson, A. Cowley, G.V. Coyne, W. Fawley, T.R. Gull, E.A. Harlan, G.H. Herbig, F. Holden, H.S. Hudson, R.O. Jakoubek, H.M. Johnson, K.M. Merrill, F.H. Schiffer, B.T. Soifer, B. Zuckerman, Astrophys. J. 196 (1975) 179-189.

[74] R.F. Warren-Smith, S.M. Scarrott, P. Murdin, Nature 292 (1981) 317-319.

[75] P.J. Sarre, Nature 351 (1991) 356.

[76] S.J. Fossey, Nature 353 (1991) 393.

[77] S.M. Scarrott, S. Watkin, J.R. Miles, P.J. Sarre, Mon. Not. Roy. Astron. Soc. 255 (1992) 11P-16P.

[78] P.J. Sarre, J.R. Miles, S.M. Scarrott, Science 269 (1995) 674-676.

[79] H. Van Winckel, M. Cohen, T.R. Gull, Astron. Astrophys. 390 (2002) 147-154.

[80] R.J. Glinski, C.M. Anderson, Mon. Not. Roy. Astron. Soc. 332 (2002) L17-L22.

[81] R.G. Sharp, N.J. Reilly, S.H. Kable, T.W. Schmidt, Astrophys. J. 639 (2006) 194-203.

[82] N. Kameswara-Rao, D.L. Lambert, Mon. Not. Roy. Astron. Soc. 263 (1993) L27-L31.

[83] L.M. Haffner, D.M. Meyer, Astrophys. J. 453 (1995) 450-453.

[84] J.P. Maier, N.M. Lakin, G.A.H. Walker, D.A. Bohlender, Astrophys. J. 553 (2001) 267-273. 
[85] F. Salama, G.A. Galazutdinov, J. Krełowski, L.J. Allamandola, F.A. Musaev, Astrophys. J. 526 (1999) 265-273. [See also database at: http://www.astrochem.org/index.htm

[86] J. Fulara, J. Krełowski, New Astronomy Reviews 44 (10) (2000) 581-597.

[87] T. Motylewski, H. Linnartz, O. Vaizert, J.P. Maier, G.A. Galazutdinov, F.A. Musaev, J. Krełowski, G.A.H. Walker, D.A. Bohlender, Astrophys. J. 531 (2000) 312-320.

[88] G.A. Galazutdinov, J. Krełowski, F.A. Musaev, P. Ehrenfreund, B.H. Foing, Mon. Not. R. Astron. Soc. 317 (2000) 750-758, and references therein.

[89] P.J. Sarre, Mon. Not. Roy. Astron. Soc. 313 (2000) L14-L16.

[90] E. Peeters, S. Hony, C. Van Kerckhoven, A.G.G.M. Tielens, L.J. Allamandola, D.W. Hudgins, C.W. Bauschlicher, Astron. Astrophys. 390 (2002) 1089-1113.

[91] D.M. Hudgins, C.W. Bauschlicher,L.J. Allamandola, Astrophys. J. 632 (2005) 316-332.

[92] T.H. Kerr, M.E. Hurst, J.R. Miles, P.J. Sarre, Mon. Not. Roy. Astron. Soc. 303 (1999) 446-454.

[93] U.P. Vijh, A.N. Witt, K.D. Gordon, Astrophys. J. 606 (2004) L65-L68.

[94] U.P. Vijh, A.N. Witt, K.D. Gordon, Astrophys. J. 619 (2005) 368-378.

[95] F. Markwick-Kemper, J.D. Green, E. Peeters, Astrophys. J. 628 (2005) L119L122.

[96] A.J. Bakhtin, I.G. Denisov, O.N. Lopatin, Optics and Spectroscopy, 79 (1995) 711-714. 

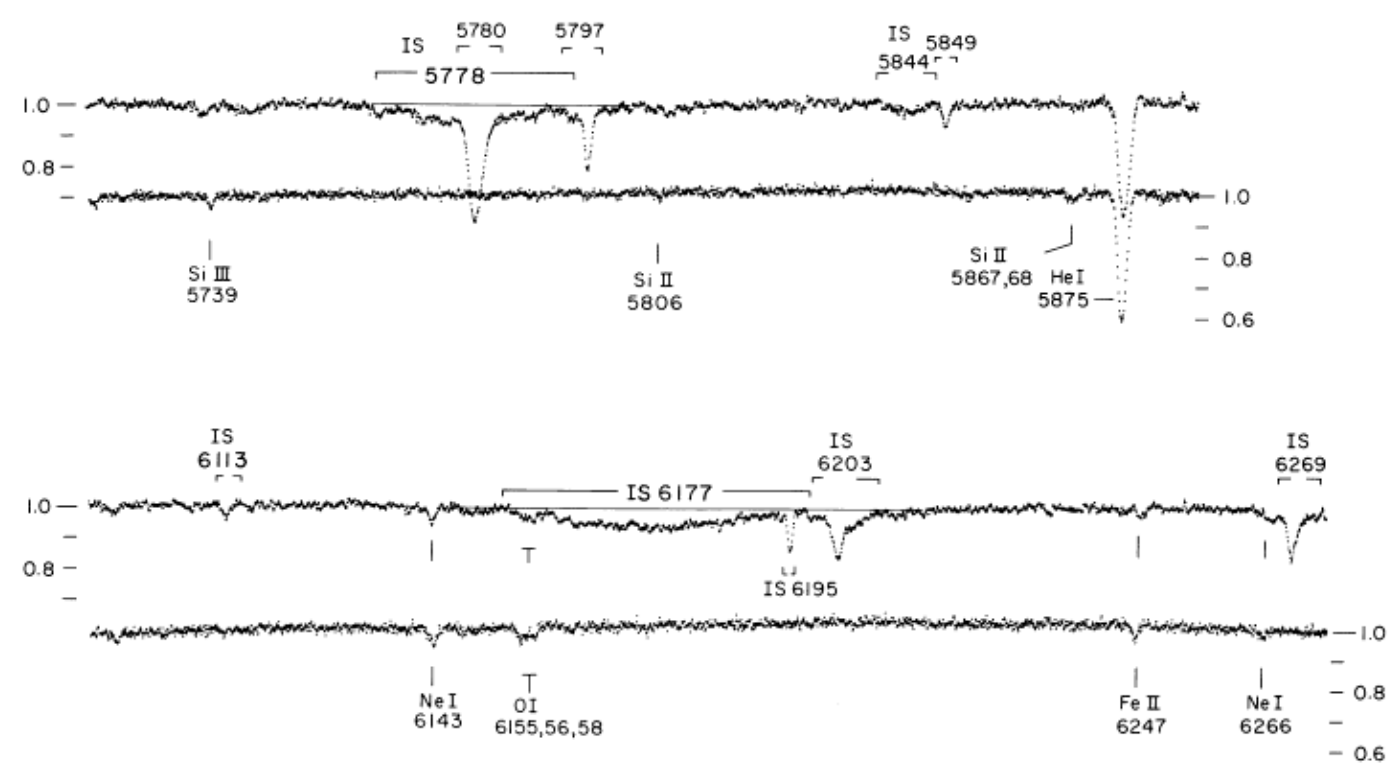

Fig. 1. Comparison of spectra towards the reddened star HD 183143 (upper of each pair of traces) with an unreddened standard (lower). IS indicates diffuse bands of interstellar origin. Note the He I photospheric line that appears in the spectra of both stars. Reproduced with permission of the AAS from [5]. 


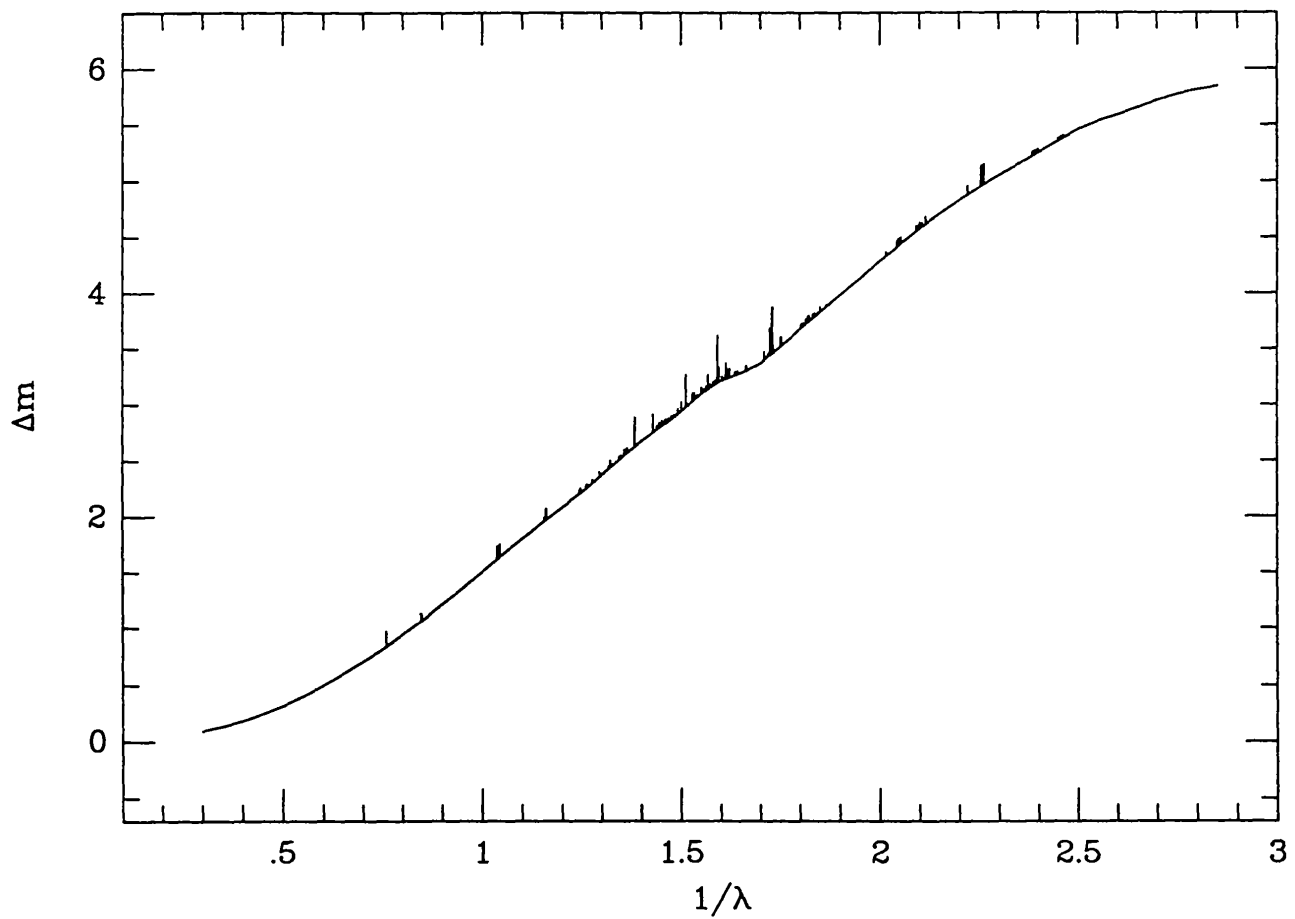

Fig. 2. Diffuse interstellar bands as 'fine structure' on the interstellar extinction curve of HD 183143. The y-axis units are magnitudes and $\mathrm{x}$-axis units $\mathrm{cm}^{-1} \mathrm{x} 10^{-4}$. Reproduced with permission from [6]. 


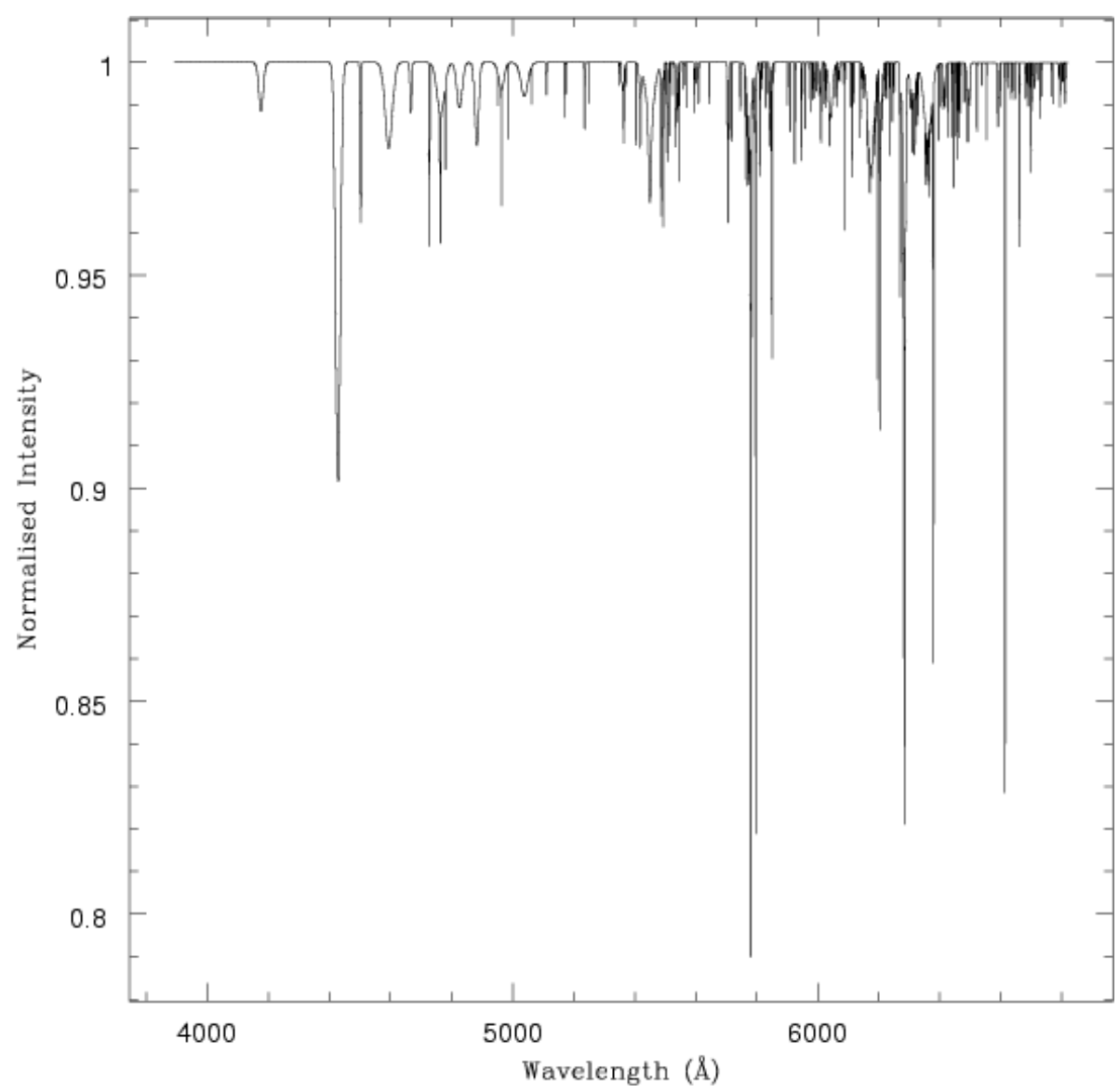

Fig. 3. Representation of 226 diffuse bands observed towards $\mathrm{BD}+63^{0} 1964$ between $3906 \AA$ and $6812 \AA$. Reproduced with permission from [14]. 


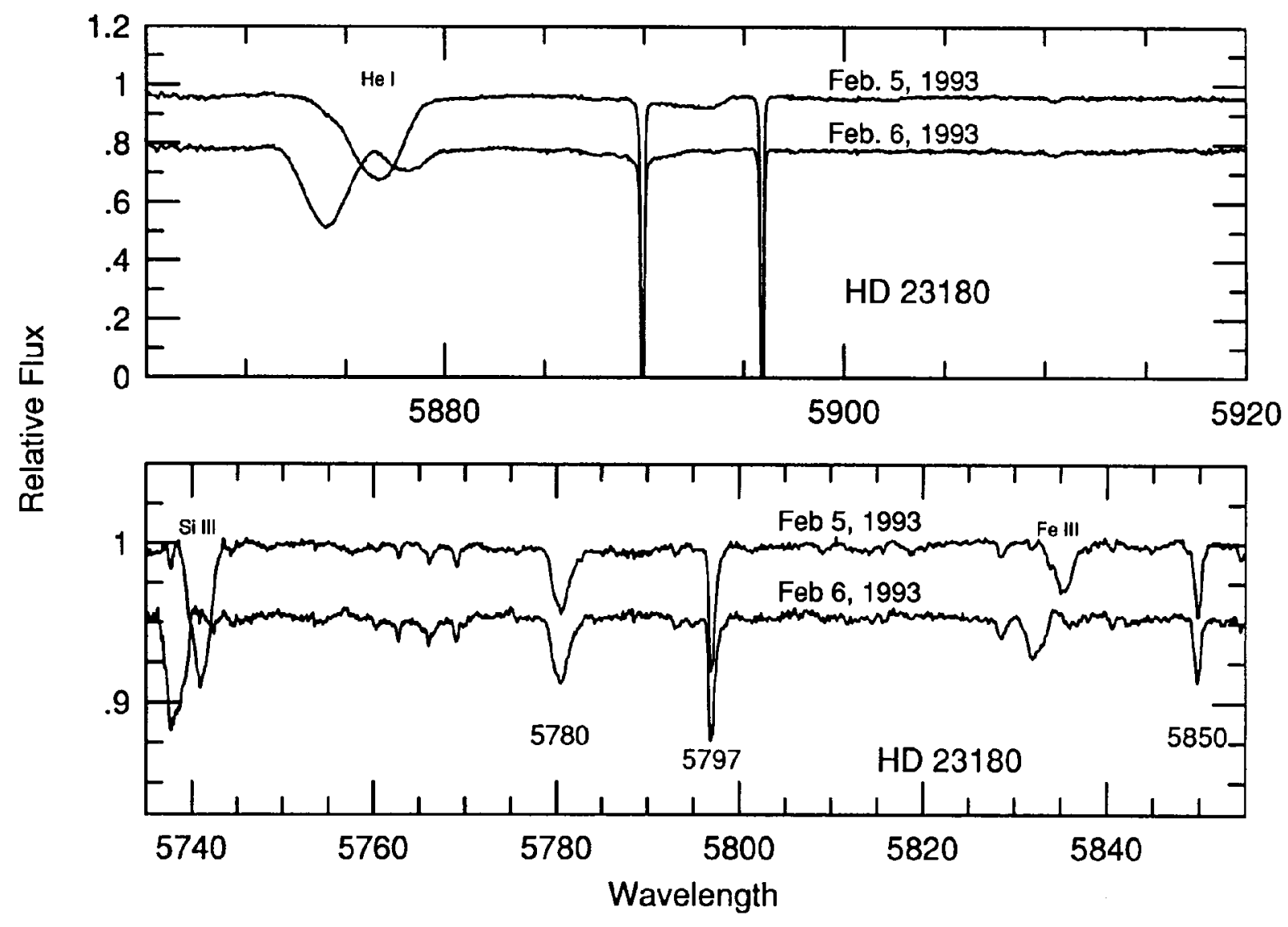

Fig. 4. Spectra of the binary HD 23180 recorded on successive nights. The stellar He I, Si III and Fe III lines are Doppler-shifted whereas the diffuse bands and $\mathrm{Na}$ I lines are stationary. This figure originally appeared in the Publications of the Astronomical Society of the Pacific (J. Krełowski \& C. Sneden, 1993, PASP, 105, 1146). Copyright [1993], Astronomical Society of the Pacific; reproduced with permission of the Editors. 


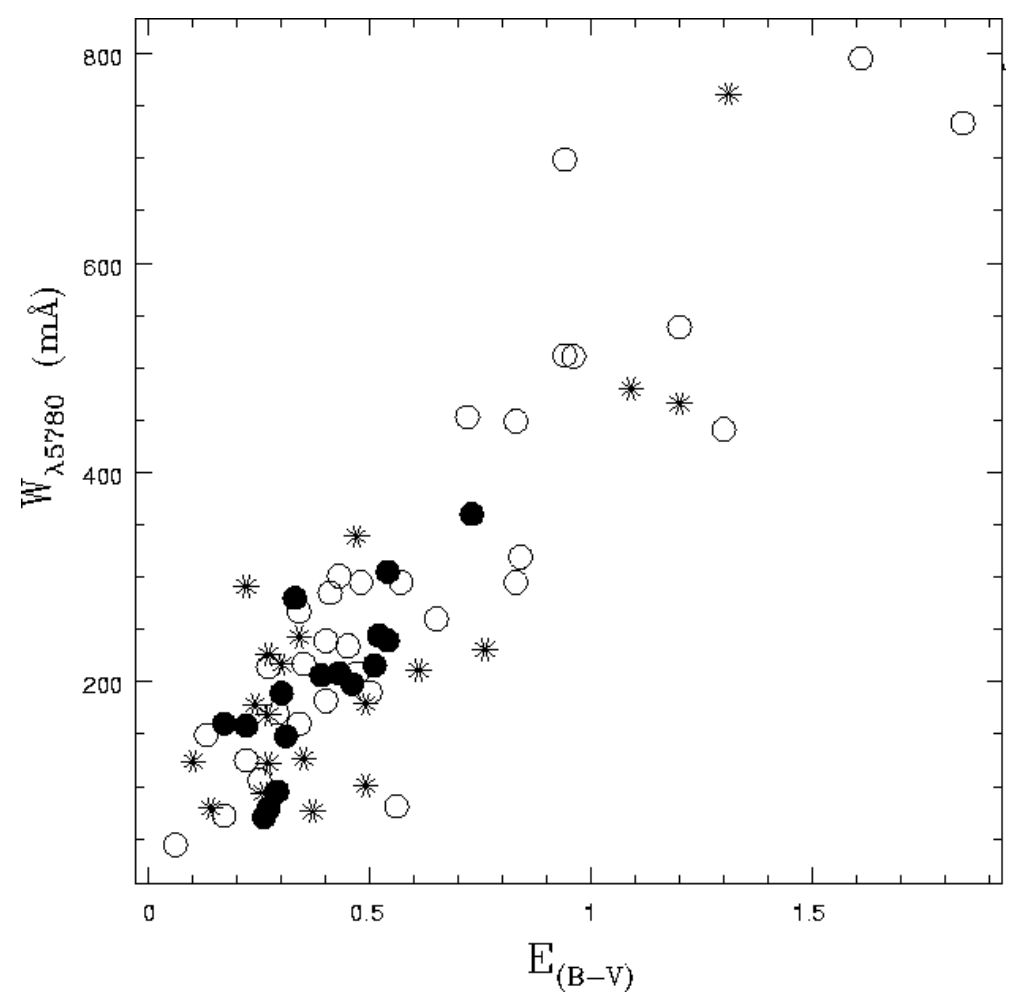

Fig. 5. Plot of the equivalent width $W_{\lambda}$ of the $\lambda 5780$ diffuse band vs. $E_{B-V}$ where the three symbols each indicate the average of different observations (see [23] for details). Reproduced and adapted with permission from [23]. 


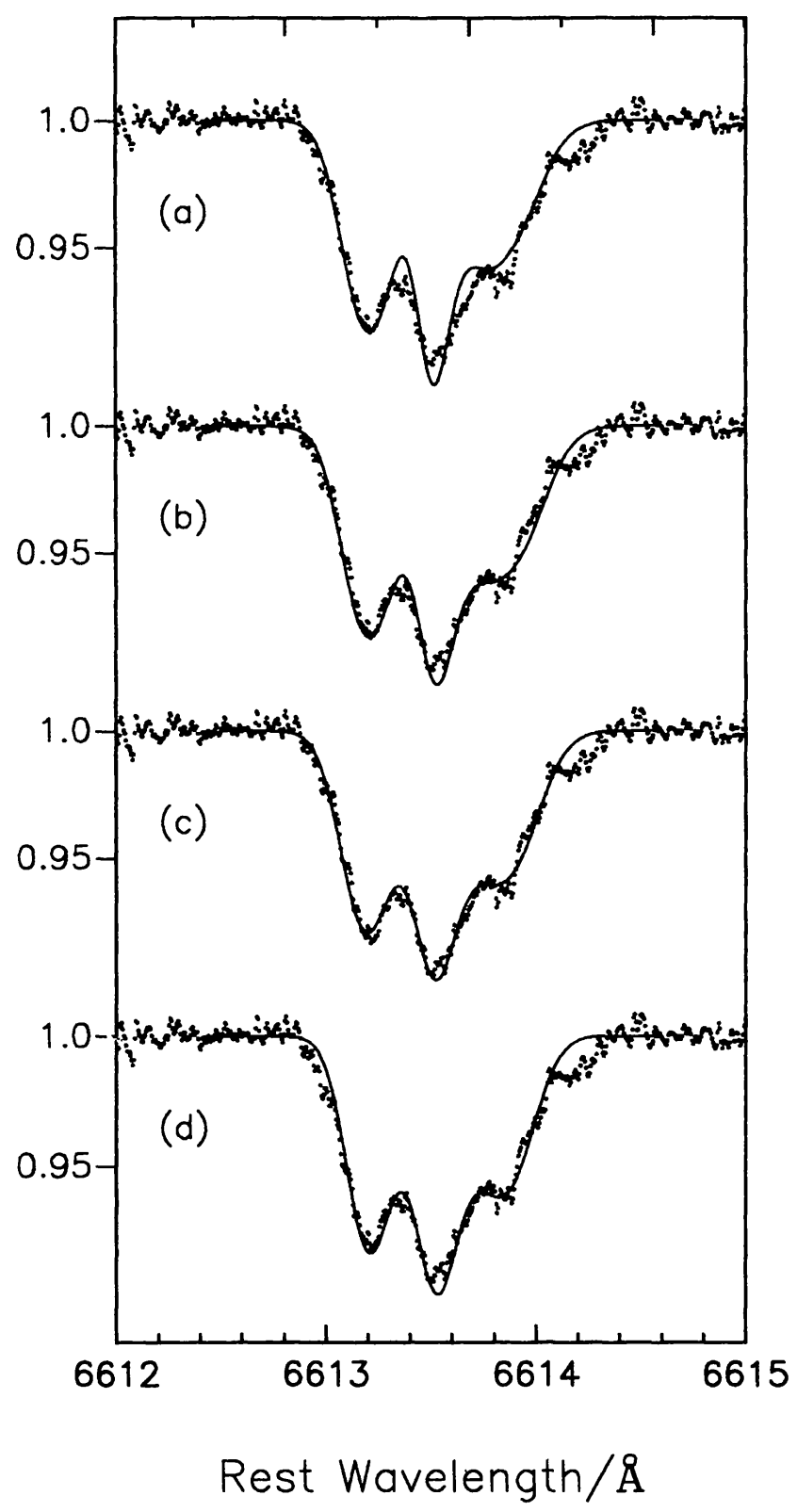

Fig. 6. Ultra-high resolution spectrum of the $\lambda 6614$ diffuse band showing fine structure. The solid lines in figures (a) to (d) are fits from different starting parameters corresponding to a range of molecular size/rotational temperature $(\sim 10-100 \mathrm{~K})$ combinations. See [52] for details. Reproduced with permission from [52]. 


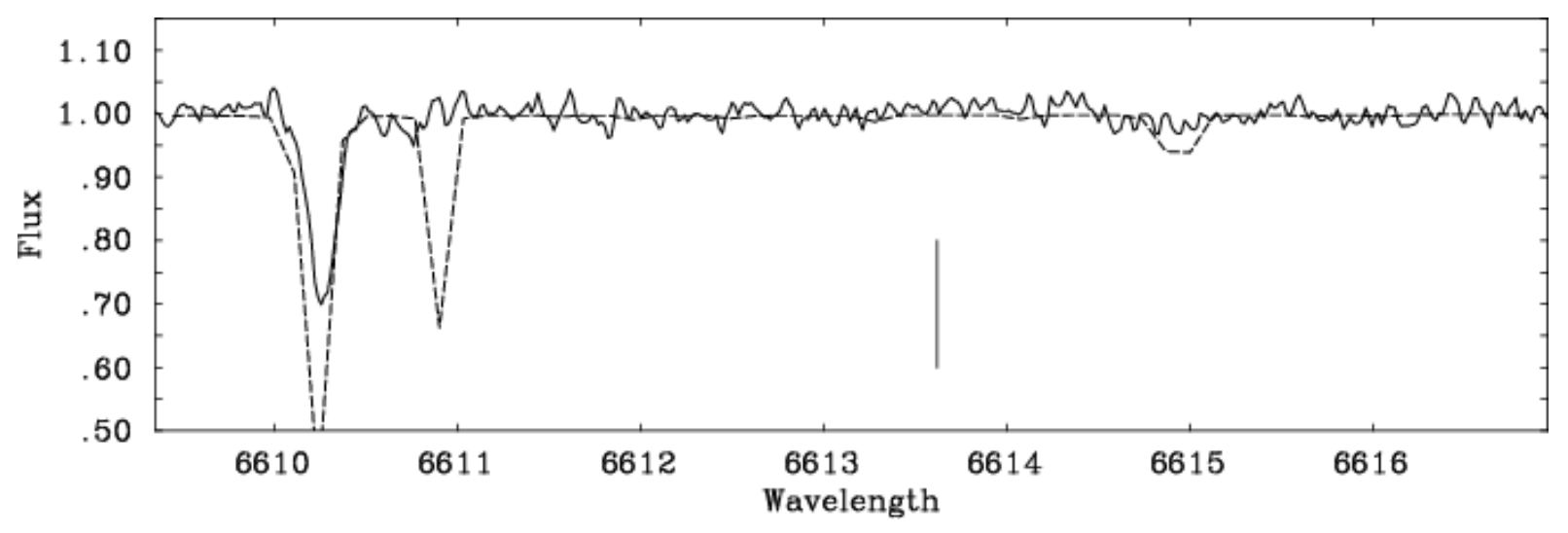

Fig. 7. Spectrum taken towards Star 6 which lies behind the circumstellar shell of IRC $+10^{\circ} 216$. No absorption at the expected position for $\lambda 6614$ is found. The feature on the left hand side is a stellar line and the dotted line is a synthesised spectrum. Reproduced with permission from [72].

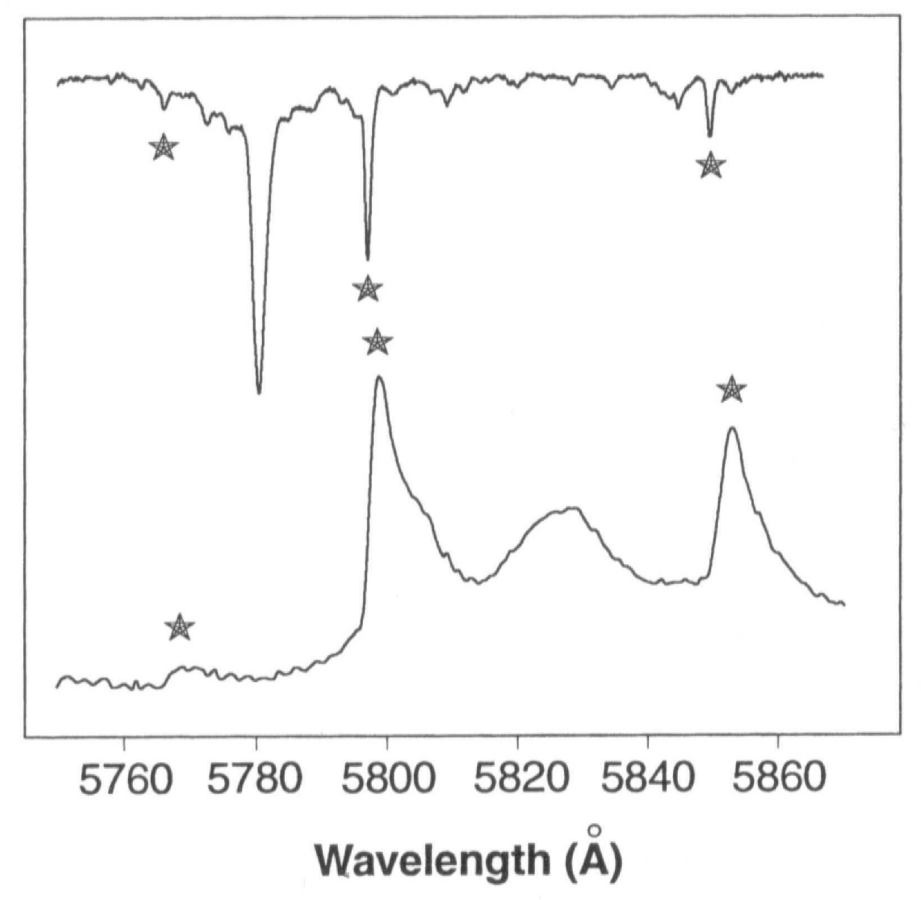

Fig. 8. Comparison between the diffuse band absorption spectrum towards HD 183143 (upper) and the Red Rectangle emission spectrum (lower). Reproduced with permission from [78]. 


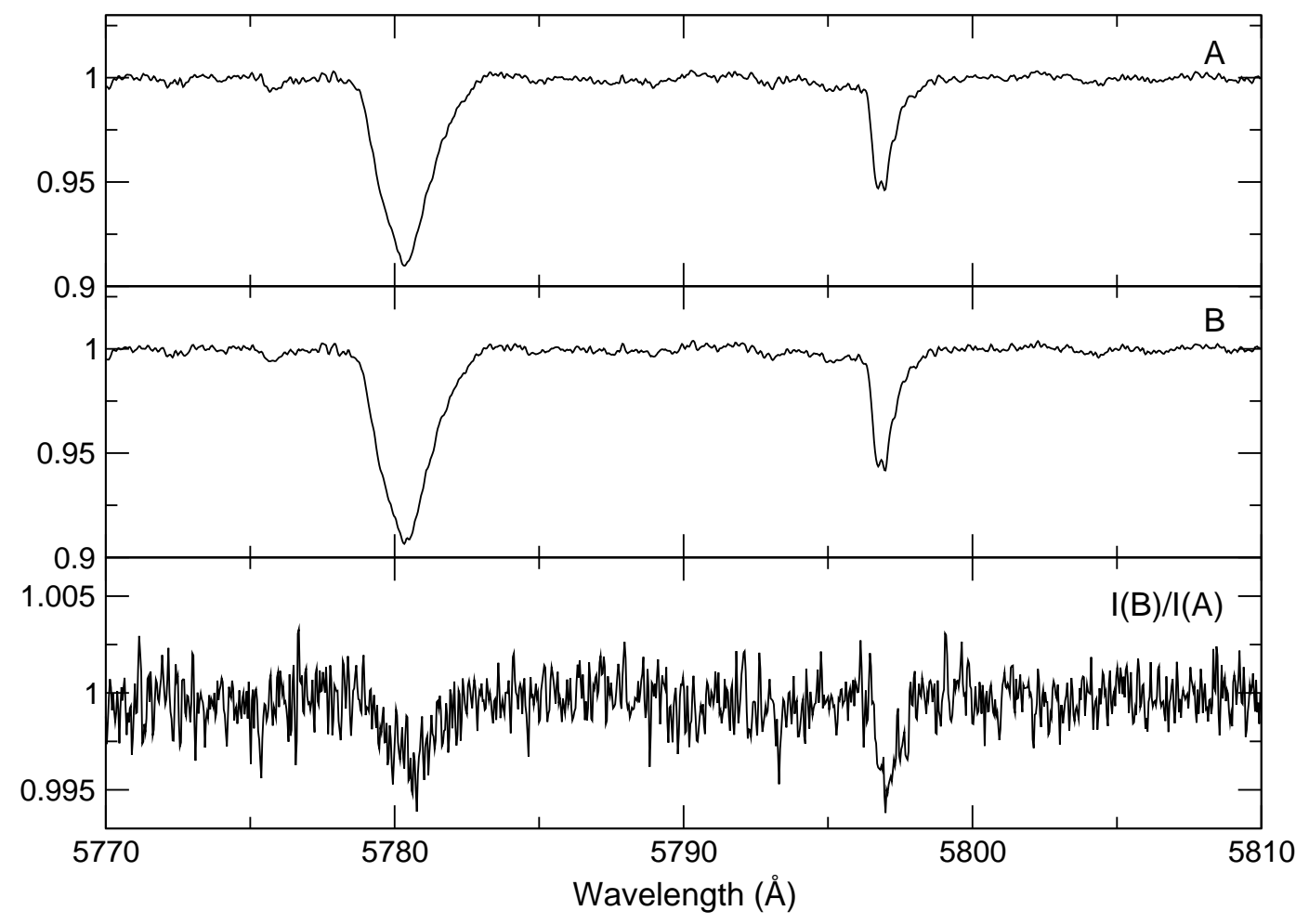

Fig. 9. Upper trace: spectrum in the region of the $\lambda 5780$ and $\lambda 5797$ diffuse bands towards $\rho$ Oph A; middle trace: spectrum towards $\rho$ Oph B; lower trace: the ratio $\mathrm{I}_{\lambda}(\mathrm{B}) / \mathrm{I}_{\lambda}(\mathrm{A})$ of the spectra towards $\rho$ Oph A and $\rho$ Oph B. The diffuse bands are stronger towards B by c. $5 \%$. 